\title{
Simplifying the propagation environment representation for LMS channel modelling
}

\author{
Mehdi Ait-Ighil ${ }^{1,2^{*}}$, Joel Lemorton ${ }^{1}$, Fernando Pérez-Fontán ${ }^{3}$, Frederic Lacoste ${ }^{2}$, Geraldine Artaud ${ }^{2}$, \\ Christophe Bourga ${ }^{4}$ and Michel Bousquet ${ }^{5}$
}

\begin{abstract}
A deep knowledge of all propagation effects has become an essential issue to design future communication and navigation systems and optimise their performances. Here, we will target Land Mobile Satellite (LMS) systems, with focus on Global Navigation Satellite Systems (GNSS). The urban environment is one of the most critical for LMS systems, since shadowing, multipath fading and time spreading are often present. This study aims at developing more efficient propagation channel models using physical-statistical approaches. In order to build these models, numerical asymptotic tools are to be used to avoid costly extensive measurements. These tools are theoretically valid for large objects. So, it is necessary to know which level of simplification of the environment is acceptable. Thus, this article performs a rigorous analysis of the influence of small scatterers at different levels of the transmission channel (up to the GNSS receiver), using the exact Method of Moments technique as a reference.
\end{abstract}

Keywords: LMS propagation channel modelling, building scattering, GNSS, Method of Moments, physical optics

\section{Introduction}

Over the past decades, mobile applications using satellites have experienced a continuous growth and the demand for such systems does not fall off. Among all developed applications, satellites have first democratised voice and message transmission and more recently multimedia broadcasting with several international standards and satellite navigation systems as Global Positioning System (GPS) and future European Galileo systems. The above systems are operating at L- and Sbands and are particularly sensitive to various channel impairments induced by urban environments, namely shadowing, multipath fading, delayed echoes, Doppler spreading, depolarisation, etc.

This study is part of a wider project aiming at reproducing channel impairments in order to build an enhanced Land Mobile Satellite (LMS) channel simulator. The future channel simulator shall be able to generate synthetic time-series for simulation needs with a specific attention on the wideband representation of the channel for Global Navigation Satellite Systems (GNSS) studies. A hybrid approach associating a virtual city [1]

\footnotetext{
* Correspondence: mehdi.ait_ighil@onera.fr

'ONERA - The French Aerospace Lab., 31055 Toulouse, France

Full list of author information is available at the end of the article
}

and a simplified physical ElectroMagnetic (EM) model has been retained. To extract such simplified EM model, numerical tools are to be used instead of extensive measurements campaigns. The tools mentioned above are based on asymptotical methods such as Physical Optics (PO) and ray-based algorithms. One of the main drawbacks of such techniques is that their validity domain is restricted to objects larger than several wavelengths. The commonly admitted limit for PO algorithm is between three to four wavelengths [2].

Due to this limitation, interactions between a complex environment with small features and EM waves cannot rigorously be modelled. The motivation of this article is to evaluate the influence of such small features to better understand what level of simplification can be applied to remain in the PO validity domain. For the present analysis, urban scenarios were addressed and especially building scattering where numerous small features are present. It also completes preliminary results presented in [3] and is mainly focusing on GNSS context. Vegetation effects and other street furniture are out of the scope of this article.

The remainder of this article is organised as follows: Section 2 presents the immediate context of this study, the retained approach and the simulation protocol we 
used. In Section 3, we present some of the results for the narrowband case which are enriched by wideband results in the GNSS context in Section 4. Finally, in Section 5, suggestions are given on what level of detail, i.e. minimum scatterer size, is required to reproduce the EM behaviour for the LMS propagation channel.

\section{Proposed approach}

\subsection{Context of the this study}

Many techniques have been used to model the LMS propagation channel where both accuracy and execution time are the key parameters. All LMS channel models usually include two main aspects: the description of the propagation environment and the modelling of EM interactions. All techniques can be grouped into three main approaches, namely statistical [4-6], deterministic and hybrid physical-statistical [7-11], depending on how the environment and the interactions are modelled. As a first step in this study, deterministic approaches are to be used before operating some algorithm simplification leading to hybrid physical-statistical models. Deterministic approaches aim at reproducing real scenarios with real EM interactions. The common factors for all methods are the realism of the environment description used as input and the use of Maxwell equations to solve the EM interaction problem. Depending on the assumptions and simplifications made, the EM initial problem will lead to different solving formulations. Deterministic approaches can be divided into two categories.

The first one covers the full-wave methods. They are directly based on Maxwell equation solutions. Being assumptions-free in their formulation and due to numerical converging criterions, those methods are said exact. The Integral Equation in Frequency Domain subcategory of full-wave methods contains Method of Moments (MoM) which will be used in this article. MoM is based on the radiation of currents induced on objects by an incident wave. The modelling can be established on surfaces or on volumes and needs boundary conditions on objects. Because of the need of large computation resources, memory and CPU efficiency, MoM and full-wave techniques are very time consuming and limited in terms of problem size to a few tens of wavelengths. In practice, those methods are not suitable at street scale and are not used for channel modelling.

The second category of deterministic approaches encompasses the asymptotic methods. Contrary to fullwave methods, asymptotic ones do not rigorously solve the EM problem resulting in time performance improvements and problem up-sizing. Asymptotic methods are particularly adapted to the channel modelling problematic. Two classes of asymptotic methods exist. The first one is based on an optical-ray assumption and includes Geometrical Optics and Uniform Theory of
Diffraction. Considering infinite sized elements, the EM field propagates along ray path satisfying Fermat's principle. The EM problem is then solved using geometric formulations such as Snell-Descartes laws. Two ray-optical algorithms are competing in the literature: the Shooting and Bouncing Rays and the Ray Tracing algorithms both presented in [12]. A second class of asymptotic methods is based on the equivalent current method and regroups Physical Theory of Diffraction and PO. Contrary to exact methods, instead of solving rigorously the EM problem, only first harmonic terms of currents are here considered using an integral formulation of Maxwell's equations. With PO-based algorithms, the main limitation is that the current assumption enounced above is valid only for objects larger than a few (e.g. three) wavelengths [2].

\subsection{Proposed approach}

Well suited for LMS channel modelling, the PO algorithm is particularly addressed in this article. Since real complex environments do not necessarily fulfil the PO validity domain, quantifying the influence of small features would permit to simplify the environment representation to satisfy PO conditions without loss of accuracy. Environment simplification could also lead to time gains considering lower complexity for the total algorithm and simpler urban Computer-Aided Design modelling.

To observe the influence of small scatterers, an adapted method has to be used, namely, MoM which does not have any restriction on object size contrary to PO. With this rigorous approach, sub-wavelength elements can be taken into consideration. In the following, we used the EM code ELSEM3D [13,14] from ONERA to compute all simulations discussed next. It is based on the Electric Field Integral Equation solved with MoM techniques in the frequency domain. Thus, this study inserts itself in a global rigorous process aiming at simplifying the channel modelling problematic from exact methods to asymptotic methods themselves reduced to physical-statistical methods in future work.

\subsection{Assumptions and simulation protocol}

As mentioned above, exact methods are resource consuming and only limited sized problems can be solved. For this reason, the whole complex urban case had to be reduced. The main element to model an urban canyon for simulation is called here a "canonical building". This canonical building represents one piece of a total building, also described as one "period" of the architectural motive. Each canonical building has a surface of about $25 \mathrm{~m}^{2}$. Still, to reduce the number of unknowns, a Perfect Electric Conductor (PEC) hypothesis has been applied to all canonical buildings. This conservative 
hypothesis does not affect the overall results presented next since they have been exploited in a relative way comparing similar simulations. Even considering those simplifications, each canonical building face presents more than 250,000 edges and needs a computation time of about $36 \mathrm{~h}$ over 8 parallel processors on a super calculator.

Regarding the PEC assumption, the reader is reminded that the interest here is on the level of simplification possible. Model parameterisation for more realistic materials (lossy dielectrics) will be performed at a later time, after having determined the level of simplification to be used and is out of the scope of this article.

To avoid loss of generality and better reflect urban diversity, the analysis has been carried out considering two types of buildings present in Toulouse, France: (1) a city centre building representative of a very "rough" surface; (2) an office building, smoother than the previous one, with only a limited number of protruding and receding elements. Both are presented in Figure 1. To evaluate the impact of small scatterers/façade features,

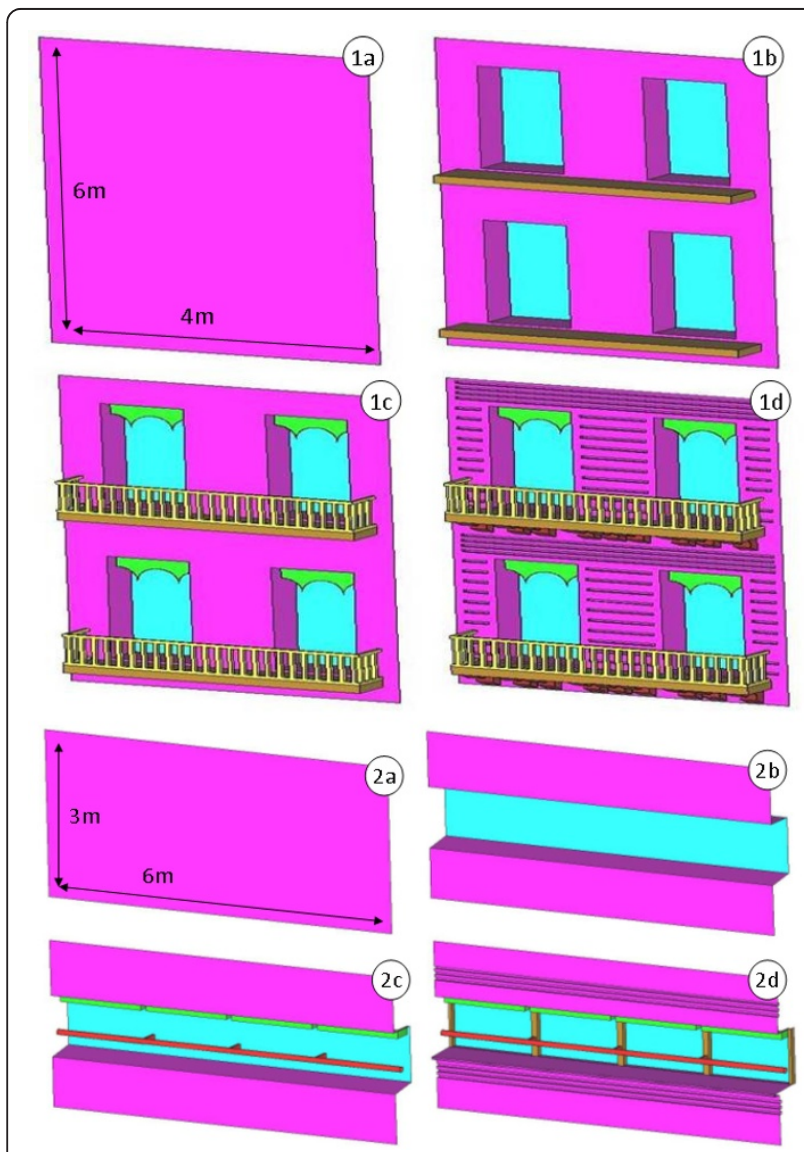

Figure 1 Representation of two canonical buildings using four resolutions. Toulouse buildings types \{(1) City centre, (2) Office\}. Environment resolution \{(a) null, (b) low, (c) medium, (d) high\}. those two canonical buildings have been reproduced four times using four different levels of detail as described next and in Figure 1:

(a) "Null" level: corresponds to a canonical plate.

(b) "Low" level: considers details much larger than one wavelength, i.e. windows and balconies.

(c) "Medium" level: considers details in the range of the wavelength, i.e. guardrails and shutters.

(d) "High" level: considers sub-wavelength details, i.e. architectural motives in walls such as bricks.

Note that this progressive description principle has also been used for terrestrial applications for indoor [15] and outdoor [16] environments.

To generalise observations made on isolated canonical buildings, more complex scenarios have been built associating several canonical buildings to reproduce urban canyons. In the following, and particularly for statistical purposes, three urban canyons have been used avoiding singular propagation effects due to particular building positioning. The geometry of Canyon 1 is the only one illustrated in this article. The Canyon 2 is similar to Canyon1, but slightly longer with a different repartition of canonical buildings into the scene. The canyon3 represents continuous façade of 80 -m-long for 12-m-hight composed of both office building type and city centre building type. Keeping in mind the GNSS context, the channel has been assessed using different speeds: 5, 50 and $90 \mathrm{~km} / \mathrm{h}$. Those speeds will have an influence on the averaging process taking place in GNSS receivers during signal integration. To reproduce this process, we used a sliding window as mentioned in Section 3.2. The assumed integration time is $20 \mathrm{~ms}$ [17] corresponding to one standard C/A GPS chip [18].

All canonical buildings described in Figure 1 have been illuminated with plane waves. A total of nine distinct incidences have been used, combination of three azimuths $\left\{0^{\circ}, 40^{\circ}, 80^{\circ}\right\}$ and three elevations $\left\{0^{\circ}, 40^{\circ}, 80^{\circ}\right\}$. To allow a global interpretation, the incidences were grouped into two different sets: "typical LMS incidence" for elevations and azimuths smaller or equal to $40^{\circ}$ (four cases) and "extreme LMS incidence" for larger elevations or azimuths (five cases). The source was linearly polarised, $\theta$ and $\phi$, at a frequency of $1.5 \mathrm{GHz}$. The $\theta$ polarisation represents a generalised case of vertical polarisation for non-null elevations while the $\phi$ polarisation represents horizontal polarisation.

All statistical analysis made in the following are based on 81 different cases for each 4 resolution representatives of the 3 different canyons, the 3 speeds and the 9 geometrical configurations in the case of a fixed $\theta$ polarisation. 


\section{Narrow band power analysis}

We now perform in this section a narrow band analysis. The purpose is to quantify the contribution of small façade features in the total multipath (MP)/scattered power. Those results would be useful when estimating, for example, the scattered power in models such as that of Oestges et al. [7] where tuning of Ricean distributions is required. First, the specific contribution of each part of one canonical building is detailed. Then, urban scenarios have been analysed associating the contributions of several canonical buildings to evaluate the MP scattered power in more complex environments. A statistical analysis follows in order to better estimate the impact of smaller scatterers.

\subsection{Specific scatterers contribution}

The goal here is to isolate and quantify the contribution of specific details into the total MP scattered power. Those results have been produced following two steps. The first step consists in using ELSEM3D to illuminate canonical buildings with the highest level of detail, (1d) and (2d) from Figure 1, and to record the induced currents over the whole surface. The second step consists in reradiating only the currents from specific parts of the canonical buildings up to the observation point. Thus, making each part of the building reradiate one after the other, the radiations are separated and each one can be isolated from the total radiation pattern. Note that the currents on each detail are calculated once all together at the first step, including neighbouring interactions, and neither discontinuity nor side effects occur when they reradiate separately. It has been verified that the coherent sum of all elementary contributions from the canonical building is equal to the total radiation when the parts are radiating all together.

The details have been grouped into four classes as defined and presented in Figure 2. The contribution of each class of detail has been evaluated in the far field. To reduce calculation time, only one quarter of the total space is considered, positive $X$ and negative $Z$ using the axis reference in Figure 2. The other quarters of space do not have any interest in our study for two reasons: negative $X$ is representative of transmission through walls which is not an issue in our case and positive $Z$ would imply that the receiver can be reached after a reflection on a wall below its horizontal plane. In practice, this last assumption reduces the observation domain since the receiving antenna diagram is normally hemispherical and oriented towards the sky, strongly attenuating the contribution from below its horizon [17].

The studied reradiating space is split into two parts: the specular zone and the non-specular zone. The

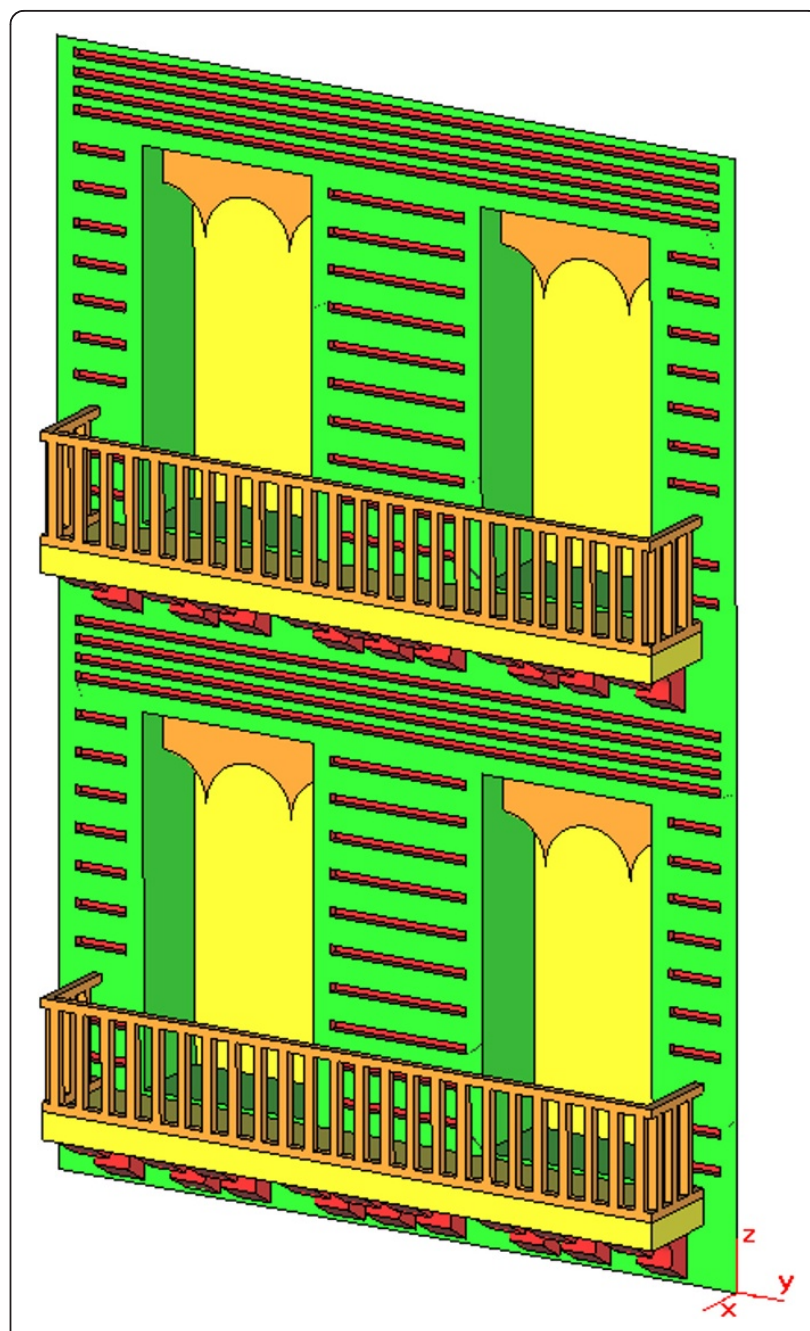

Figure 2 Description of the 4 different classes of details. Class 1, green, contains only the flat parts of the walls. Class 2, yellow, contains windows and balconies. Class 3, orange, contains shutters and guardrails. Class 4, red, contains all smaller details. Each class of detail roughly reproduces the characteristics of each level of detail.

specular zone is defined by $\pm 2^{\circ}$ around the geometric specular point. In the results presented next, we only consider the magnitude of the scattered electric field without considering any polarisation loss at the receiver. The contributions in Table 1 are presented in unit scale. One unit represents the total MP scattered power using a high-resolution model. Note that the sum of each line equals one.

From Table 1, the scattered power received in the specular zone mostly comes from the flatter parts of the wall, $50 \%$ in the presented case. The contribution of the smaller details is almost negligible in this zone, about $4 \%$. However, in the non-specular zone, the contribution of the small details increases, reaching $12 \%$ of the total MP scattered power, while the contribution of the flat 
Table 1 Contribution of each class of details for specular and non specular zones for city centre building type in configuration $40^{\circ}$ elevation, $0^{\circ}$ azimuth, $\theta$ polarisation

\begin{tabular}{lllll}
\hline Unit & Flat walls (green) & Window balcony (yellow) & Guardrail shutters (orange) & Small details (red) \\
\hline Spec & 0.50 & 0.21 & 0.25 & 0.04 \\
N. spec & 0.25 & 0.34 & 0.29 & 0.12 \\
\hline
\end{tabular}

parts decreases. Also noticeable is the contribution of the intermediate details, columns 2 and 3, which increases in the non-specular zone compared to the specular zone. As expected, the above observations confirm the fact that large and flat surfaces dominate in the specular zone while the smaller objects and sub-wavelength details are dominant out of the specular zone.

The results from Table 1 have been generalised for multiple incidences using both $\theta$ and $\phi$ polarisations over city centre type and office type buildings. Figure 3 presents the generalised results in the case of a city centre building type for $\theta$ polarisation. Note that the Radar Cross Section (RCS) coefficient is given for each incidence angle to provide a better idea of the physical quantities involved. RCS has been evaluated only at the specular point. As a comment, it is noticeable that the RCS decreases with the angle due to projection and visibility effects from the source to the facade. As a comparison, RCS of a metallic plate of this size would be $52.57 \mathrm{dBm}^{2}$ for normal incidence and $47.94 \mathrm{dBm}^{2}$ in the

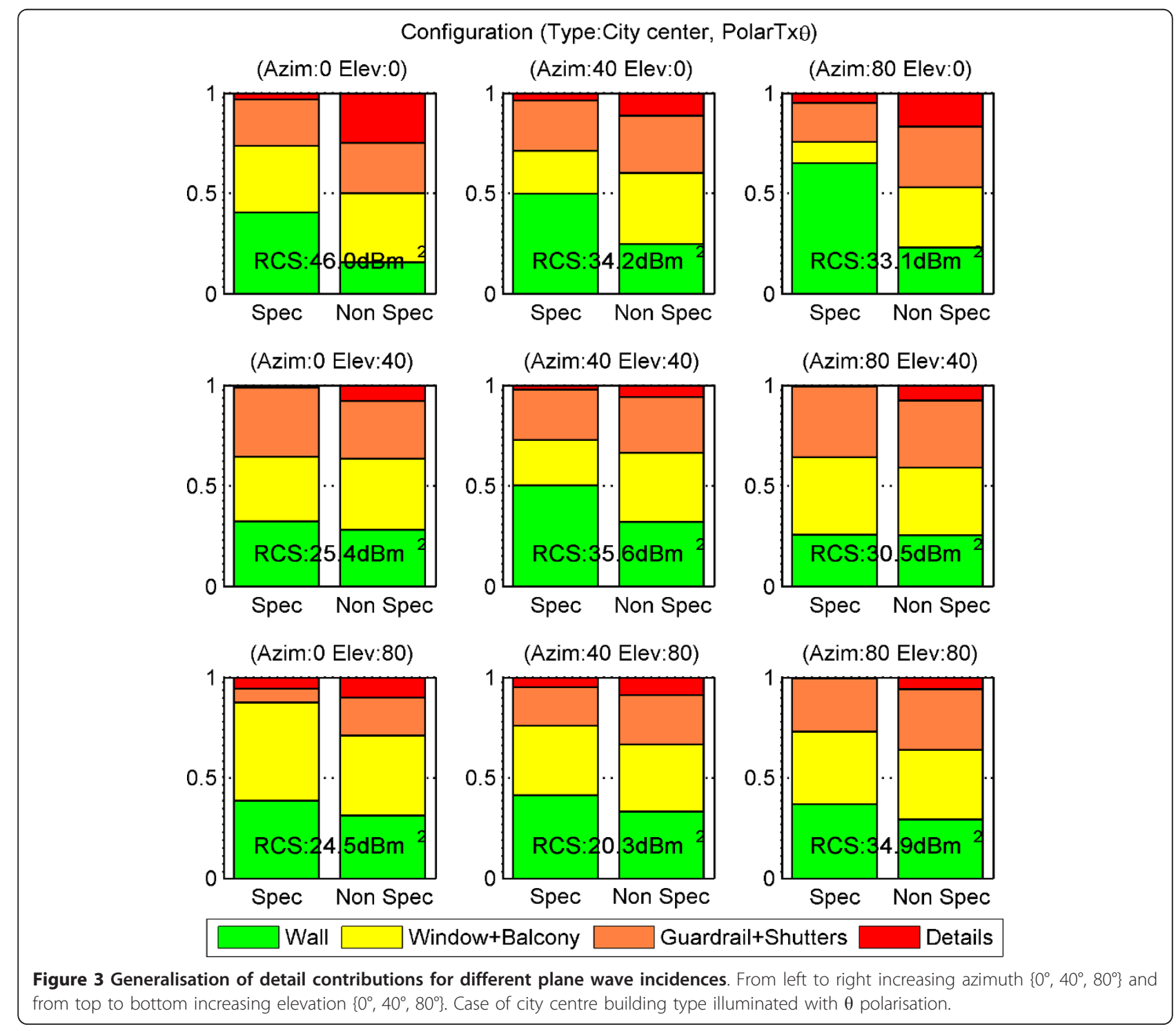


case of $40^{\circ}$ elevation, $40^{\circ}$ azimuth. In Figure 3, comparing RCS values, it is noticeable that all details contribute to slightly decrease the total RCS in the specular zone. Figure 3 also reveals that the details in the range of one wavelength and smaller, classes 3 and 4, have a greater impact out of the specular zone. Those details, present in medium and high resolutions, reradiate 30 to $50 \%$ of the total scattered power out of the specular zone depending on the geometrical configuration. In the specular zone, the relative contribution of the same details is lower.

The conclusion is that details are more visible out of the specular zone even if their absolute contribution is not higher. Small details tend to scatter power in every direction almost uniformly. In the specular zone, those small contributions are masked by the strength of the specular reflection.

\subsection{Multipath scattered power in urban scenarios}

The goal here is to estimate the influence of the resolution used on synthesised scattered power time series. In this case, representative urban scenarios, as described in Section 2.3, have been created using multiple canonical buildings. To generate time series a single contribution has been assumed from each canonical building. The scattered power is represented here relative to the unblocked direct signal.

The assumed scenario is as follows: a receiver is moving along a linear trajectory parallel to a row of buildings where each may be made up of various canonical elements. Varying the buildings' façade resolutions, the obtained time series differ. It is this difference, in terms of narrowband MP scattered power that is analysed below to quantify the error made when simplifying the environment with respect to the high-resolution case.

To produce complex time series, the reflected EM field has been calculated using ELSEM3D along one trajectory for the two canonical buildings, the four resolutions, the three elevations, the three azimuths and the two polarisations. The receiver trajectory is parallel to the $y$-axis and is sampled every $\lambda / 10 \mathrm{~m}$ over a range of $\pm 300 \mathrm{~m}$ around building centre. This $300 \mathrm{~m}$ limit corresponds to the path difference beyond which multipaths will not interfere with the direct signal to introduce pseudo-range errors in GNSS receivers as it will be eliminated by the Delay Lock Loop (DLL) [17].

For more realism, the EM field has been calculated considering three other possible locations of the canonical elements as shown in Figure 4. This results in a database taking into consideration two floors $(z=0, z=$ $+3 \mathrm{~m}$ for office buildings or $z=+6 \mathrm{~m}$ for city centre buildings) and two rows of buildings ( $x=0 \mathrm{~m}, x=-6$ $\mathrm{m})$, different back settings from the road. The total MP scattered power was summing coherently all contributions at the receiving point, as presented in Figure 4. Canyon1 is one example of urban environment used in the following (see Figure 5). Note that only one side of

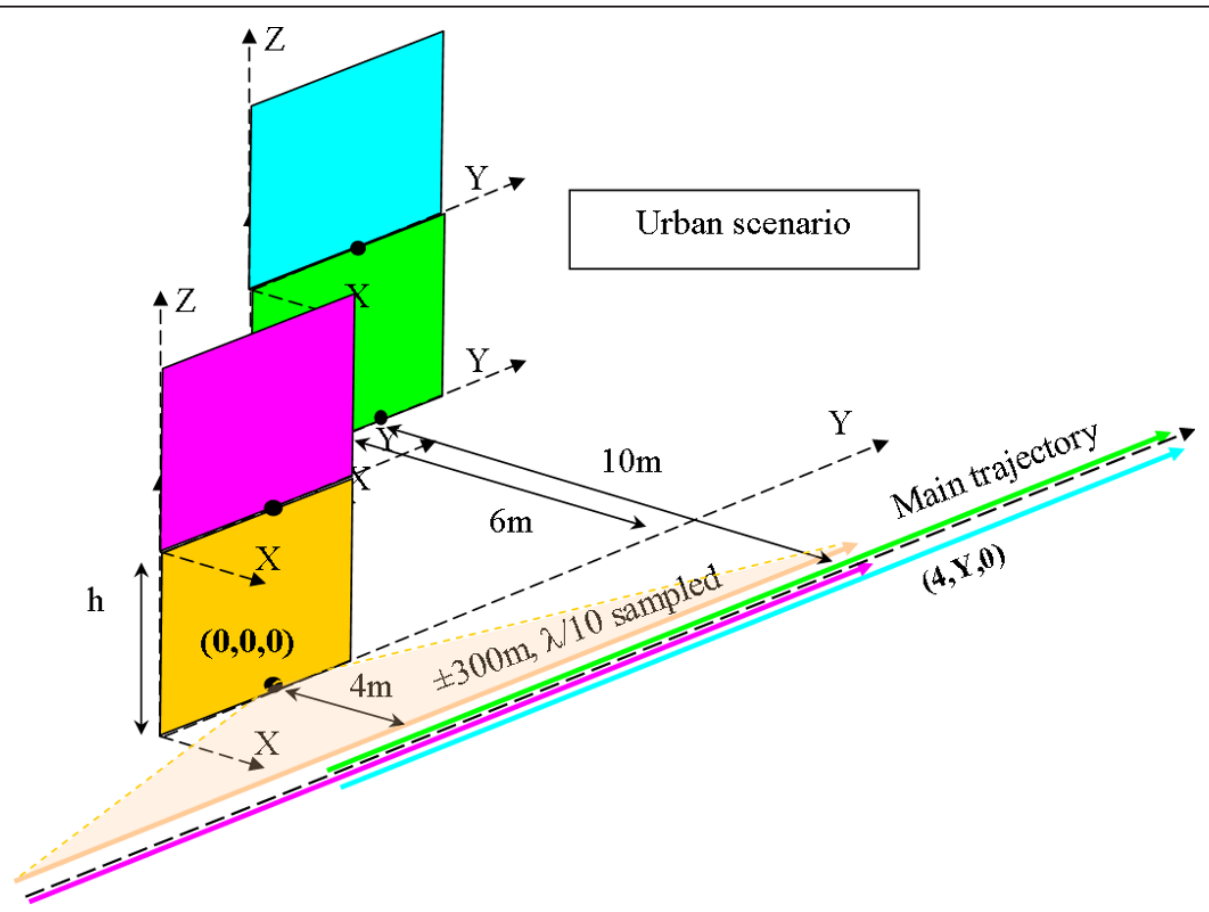

Figure 4 Summing contribution principle for complex urban scenarios generation. 


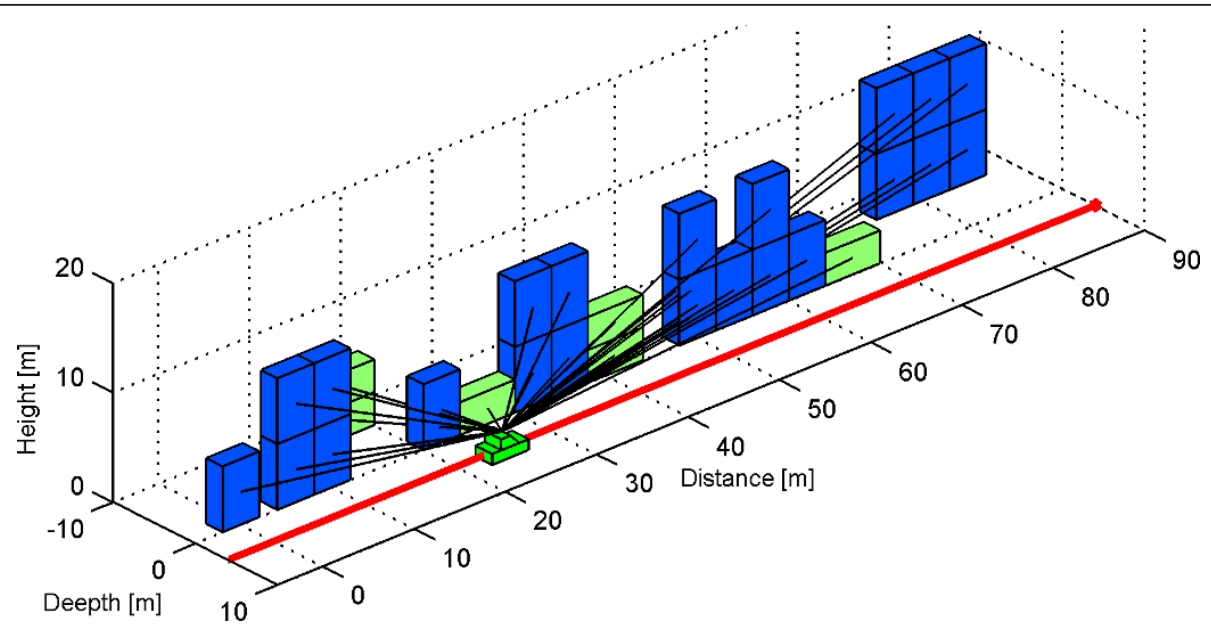

Figure 5 Example of perspective view for urban environment: Canyon1. The receiver is following the red line through the canyon. Blue buildings are city centre type and green buildings are office type. The resolution parameter is applied to the whole scenario.

the street is represented, the other one being building free.

Figures 6 and 7 show the MP scattered power time series for Canyon 1 in two different configurations. Figure 6 represents a standard geometrical configuration for LMS applications while Figure 7 illustrates the case of higher elevation of the source. At the top of both figures, the MP scattered power relative to LOS is presented as function of receiver position for each resolution. At the bottom, the relative error made when estimating the MP scattered power for simplified environments with respect to the high resolution is plotted. In the bottom graphics, the integration process of $20 \mathrm{~ms}$ has been taken into account, contrary to top ones, to better observe the result of the averaging process.

From a visual inspection of all 81 configurations, presented in Section 2.3, several statements can be made. The first is that the MP scattered power times series are very dependent on the canyon profile. The influence of each building, position and height, is visible on times series. For example in top of Figure 6 between 60 and $80 \mathrm{~m}$, the influence of the last blue building in Canyon1, Figure 5, is clearly visible. The second observation is the obvious lack of accuracy when using the null resolution compared to the high-resolution model with differences from +10 to $-30 \mathrm{~dB}$. For typical LMS incidences, below $40^{\circ}$ in elevation and azimuth, the null resolution tends to overestimate the MP scattered power in the specular zone, as shown at the bottom of Figure 6. This can be explained by the existence of specular points on facades inducing a perfect geometrical reflection on the walls. The region from -5 to $80 \mathrm{~m}$ is then characterised by the presence of strong echoes. On the contrary, in nonspecular zones, the null resolution tends to underestimate the MP scattered power. This is particularly visible for extreme incidences. Figure 7 is a typical illustration of this phenomenon. At $80^{\circ}$ elevation, specular point on facade do not exists anymore. The MP power is then highly underestimated. It is also visible at the beginning and at the end of the series in both Figures 6 and 7, when the receiver is out of the reflection zone of the canyon.

Looking at all 81 time series, the results for the low and medium resolutions show good agreement with respect to the high-resolution case and it remains difficult to discriminate which one is accurate enough. Thus, to better compare them, a statistical analysis has been conducted. From each simulated configuration, two error parameters were extracted from the relative MP scattered power (bottom graphics): the mean error and the standard deviation error. Both parameters have been calculated only in the reflection zone, approximately from $y=0$ to $85 \mathrm{~m}$ depending on the incidence angle, where specular phenomenon is present.

The statistics shown in Figure 8 present the influence of the environment resolution on the MP scattered power. A cumulative density function (cdf) based on all 81 configurations is here used to present both error parameters over the different resolutions. The medium resolution shows the best agreement in terms of mean error and standard deviation. However, the mean error and the standard deviation for low resolution present similar slopes. The difference between both resolutions is about $2 \mathrm{~dB}$ for the mean error and less than $1 \mathrm{~dB}$ for the standard deviation. Thus, the low resolution could be acceptable for calculating the MP scattered power. Indeed, by slightly filtering the time series to reduce their dynamic and adding an empirical offset to the obtained MP scattered power of about $2 \mathrm{~dB}$ to compensate the mean error, the low resolution could be a good 


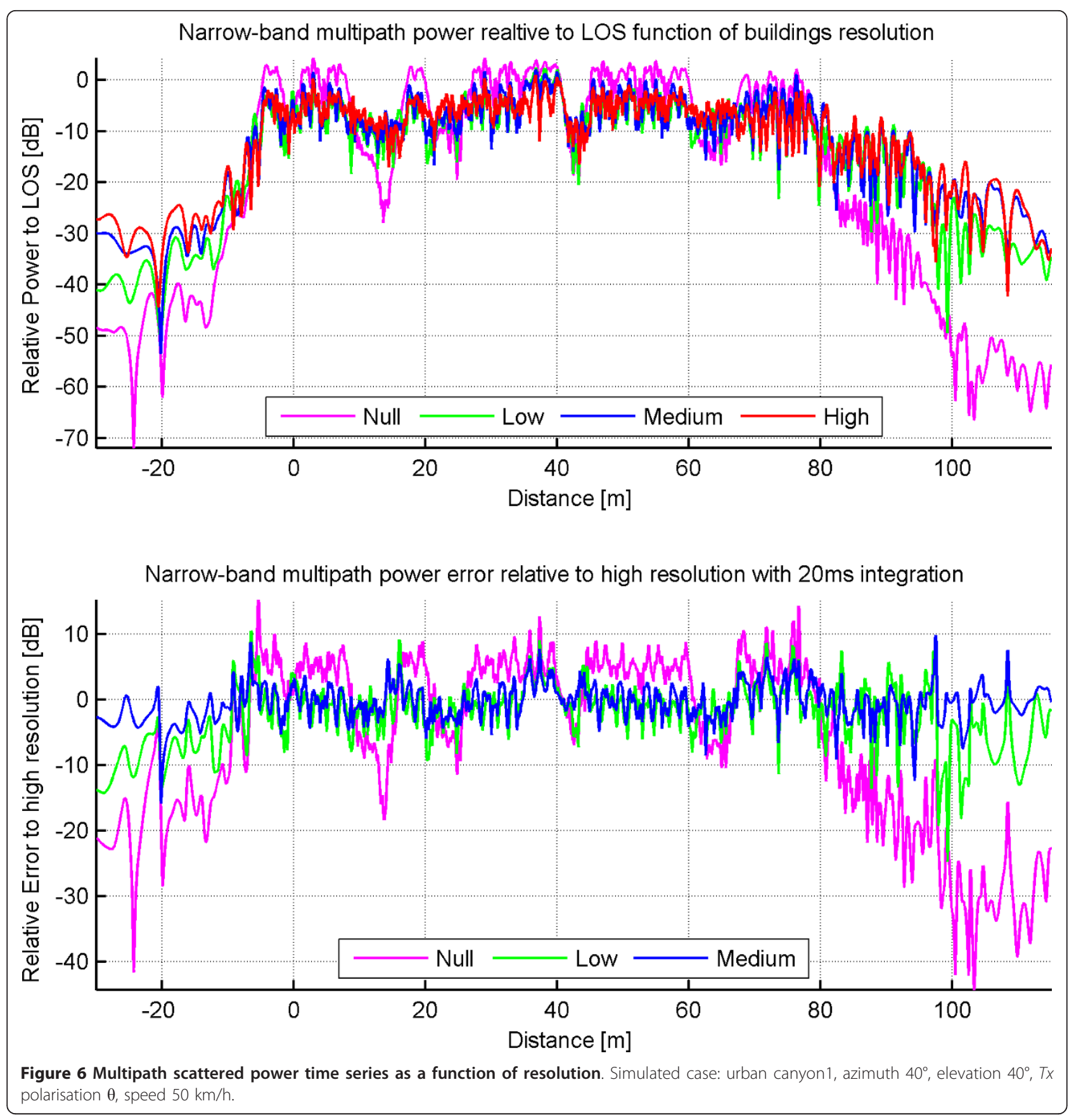

compromise to quickly obtain a good estimate of the MP scattered power. Nevertheless, a major gap can be observed between the null resolution and other resolutions. A general observation is that the mean relative errors are mostly negative for all three simplified resolutions, due to a reduction of the scattered signal when simplifying environment representation. For example, even for the medium resolution the median of the mean error is about $-0.7 \mathrm{~dB}$.
The statistics shown in Figure 9 present the influence of speed on MP scattered power. This figure has been produced using 27 cases ( 3 canyons, 1 speed and 9 geometrical configurations) considering a fixed medium resolution to avoid the influence of this parameter. This figure reveals that the speed has no impact on the mean error since all cdfs are almost equal. However, a constant difference of about $2 \mathrm{~dB}$ is observable for the standard deviation between slow motion, $5 \mathrm{~km} / \mathrm{h}$, and fast 

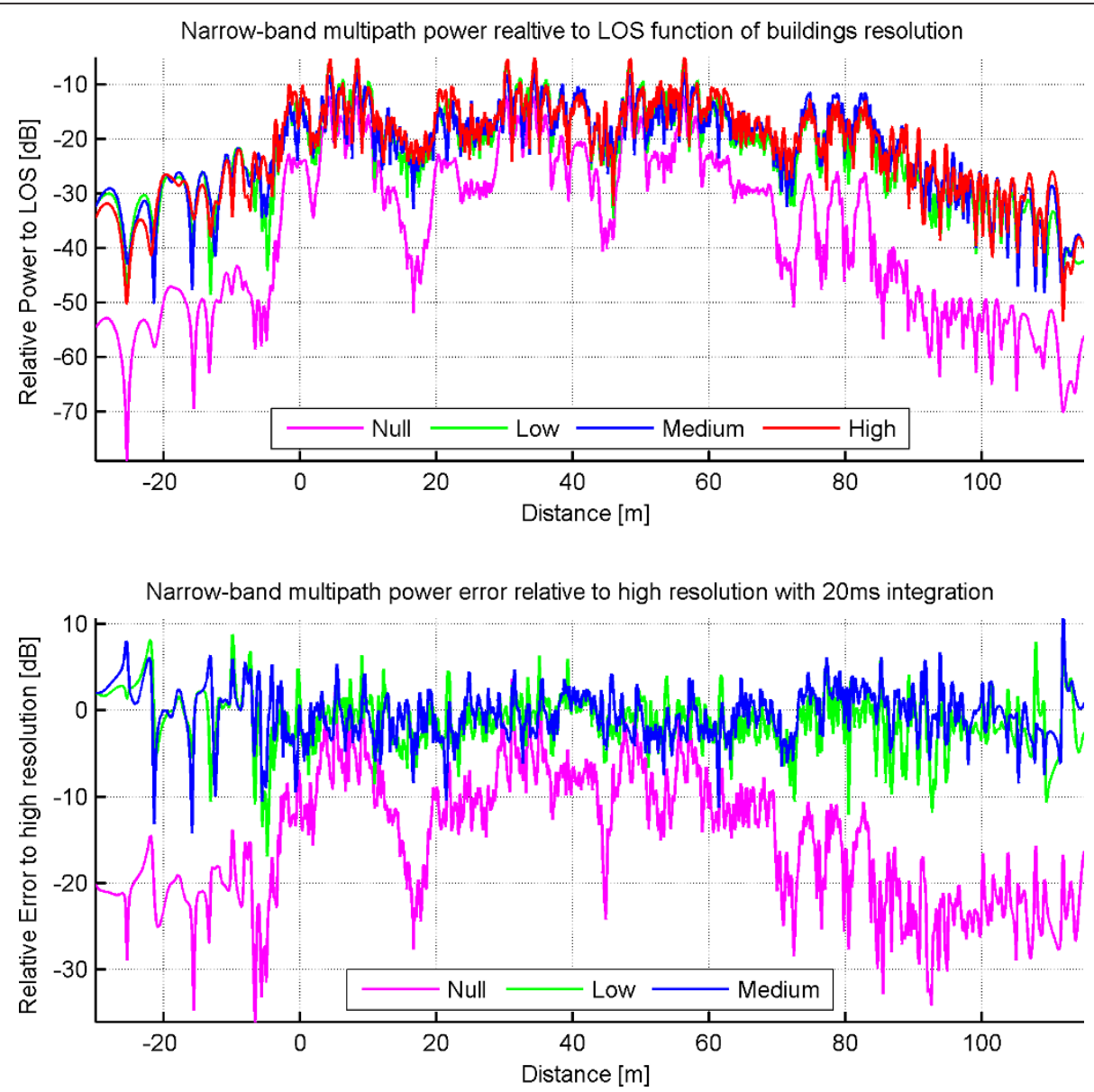

Figure 7 Multipath scattered power time series as a function of resolution. Simulated case: urban Canyon 1 , azimuth $40^{\circ}$, elevation $80^{\circ}, T x$ polarisation $\phi$, speed $50 \mathrm{~km} / \mathrm{h}$.
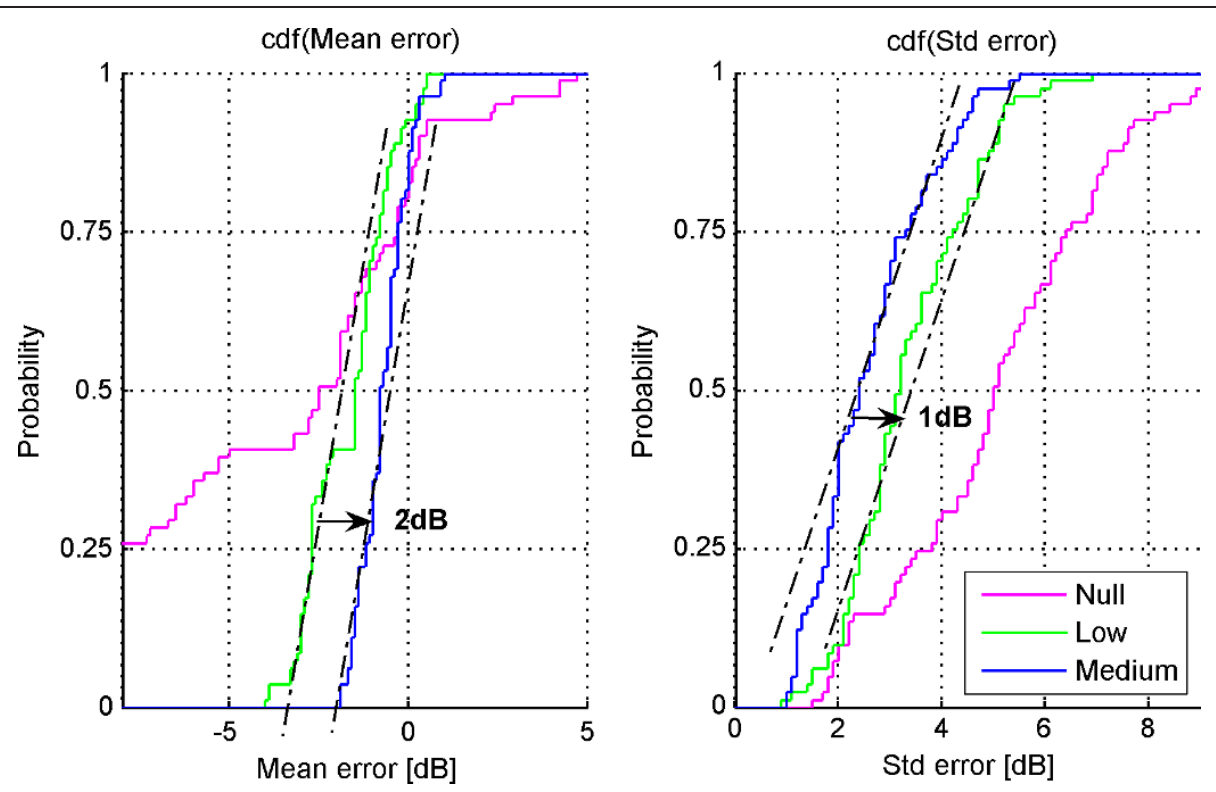

Figure 8 Influence of environment resolution on MP scattered power relative to high resolution. Left diagram presents cdf of mean error. Right diagram presents cdf of standard error. 

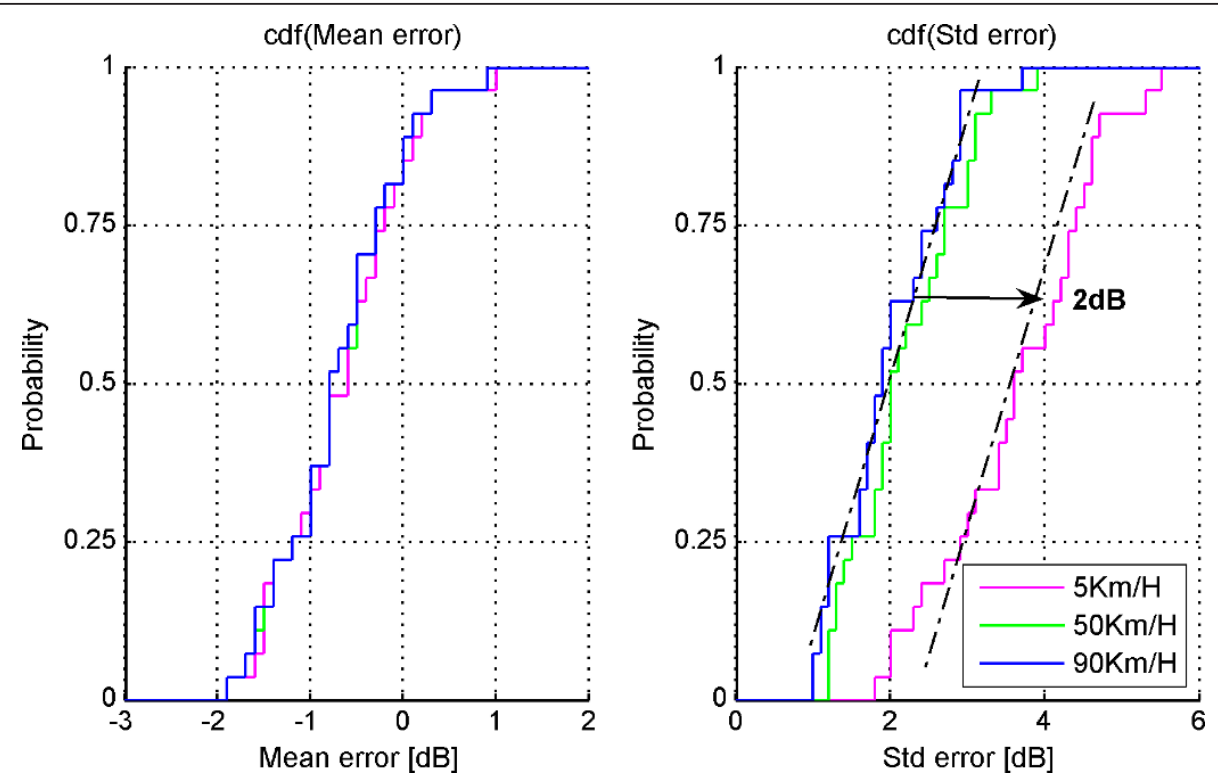

Figure 9 Influence of speed on MP scattered power relative to high resolution. Left diagram presents cdf of mean error. Right diagram presents cdf of standard error.

motion, 50 and $90 \mathrm{~km} / \mathrm{h}$. This phenomenon is due to the integration process which mitigates fading depth, decreasing signal dynamics and therefore the standard deviation of the error made.

To conclude this narrowband analysis we can make the following statements. The details in the range of one wavelength and smaller, highly contribute to the scattering phenomenon and are particularly visible where no specular reflection occurs. Neglecting them would unbalance the specular and scattering phenomena favouring the strong reflections. Adding details large compared to the wavelength, representative of the low-resolution case, would correctly attenuate the specular reflection without fully reproducing the scattering phenomenon. Results using low resolutions could be improved by adding the scattered power not accounted for, which is here in the order of $2 \mathrm{~dB}$, thus achieving comparable results to those obtained with medium resolutions.

\section{Wideband analysis}

To complete the above narrowband analysis, a wideband study was performed particularly addressing the GNSS case. The goal is to quantify the influence of the environment resolution on the receiver performance when estimating the pseudo-range to a satellite. First, the simplified GNSS receiver model used is described and then the wideband channel model used to generate the channel response is presented. Finally, a qualitative analysis of the pseudo-range estimation error is shown followed by a statistical analysis of the influence of environment resolution.

\subsection{Simplified GNSS receiver model}

The simplified GNSS receiver used in this study was developed by the French Space Agency, CNES. It aims at reproducing the various internal processes of a typical receiver in a simplified way in order to estimate pseudorange errors. The simplifications are mainly based on the fact that the receiver directly operates on post-correlation synthetic data instead of operating on baseband synthetic data. Hence, the standard C/A GPS code [18], a spreading Pseudo Random Noise (PRN) sequence, is here modelled as a simple triangular function representing the autocorrelation of the PRN code. Then, in the presence of multipath, the overall correlation function is constructed by combining the complex delayed correlation functions due to each individual MP path. The DLL is implemented as a dot-product discriminator followed by a first-order loop filter [17]. The "early" and "late" replicas of the local code are spaced 0.25 chips from the "prompt", and the DLL noise bandwidth is set to $10 \mathrm{~Hz}$. The output of the Phase Lock Loop (PLL) is emulated assuming noise-free conditions, and then, the phase noise is added to the "prompt" signal using randomly generated samples whose standard deviation corresponds to that of a Costas PLL with $1 \mathrm{~Hz}$ noise bandwidth. The tracking time is set to $20 \mathrm{~ms}$.

Two input files are required: the first contains the receiver parameters and the second is devoted to the channel characteristics. The channel file contains several parameters such as the time of week, the navigation data, the ionospheric delay or the number of MP paths. Each path, including the direct signal, is defined 
considering its power, phase, delay and Doppler. Power and phase parameters are given relative to the direct signal. The output file gathers the pseudo-range error, the tracked phase and the demodulated navigation data. Note that an acquisition step is performed by the receiver but all the simulations presented below have been realised assuming that the receiver was in the "locked" state. The thermal noise level was assumed to be -202 $\mathrm{dBW}$ and the direct signal power $-153 \mathrm{dBW}$.

\subsection{Wideband representation of the channel}

As for the narrowband analysis, the MoM-based tool ELSEM3D was used to determine the EM field along linear trajectories. The urban canyon under consideration here has been built using the same principles as in Figure 4. Contrary to the narrowband analysis where the contributions of canonical buildings were coherently summed all together, each building contribution is now represented by one ray (delta function) discriminated in terms of power, phase, delay and Doppler. The power of each ray is assumed to be equal to the narrowband power radiated by each canonical building and depends on the environment resolution as presented in Figure
10. Figure 10 permits a better understanding of how the chosen environment resolution could affect the GNSS receiver since the evolution of one specific echo has been highlighted for all four resolutions to observe its power variation along the receiver route. As visible in Figure 10, the maximum power of the red ray slightly decreases when adding details to the facade. Also visible a back scattering zone around $50 \mathrm{~m}$ where power is increasing when adding details. The phase, delay and Doppler shift are determined using a geometrical approach where rays originate from the centre of each canonical building. This construction implies that phase, delay and Doppler do not depend on the resolution employed. The power and delay of each ray are considered relative to the LOS signal. The phase and Doppler are considered in their absolute values. Note that the navigation satellite is supposed to be stationary and the observed Doppler shifts are only due to the receiver's motion. Figure 11 illustrates the evolution of the channel parameters, power, delay and Doppler, while the receiver is moving through the Canyon1. As predictable, Figure 11 reveals that the strongest echoes are the nearest. The evolution of Doppler and delay of each echo

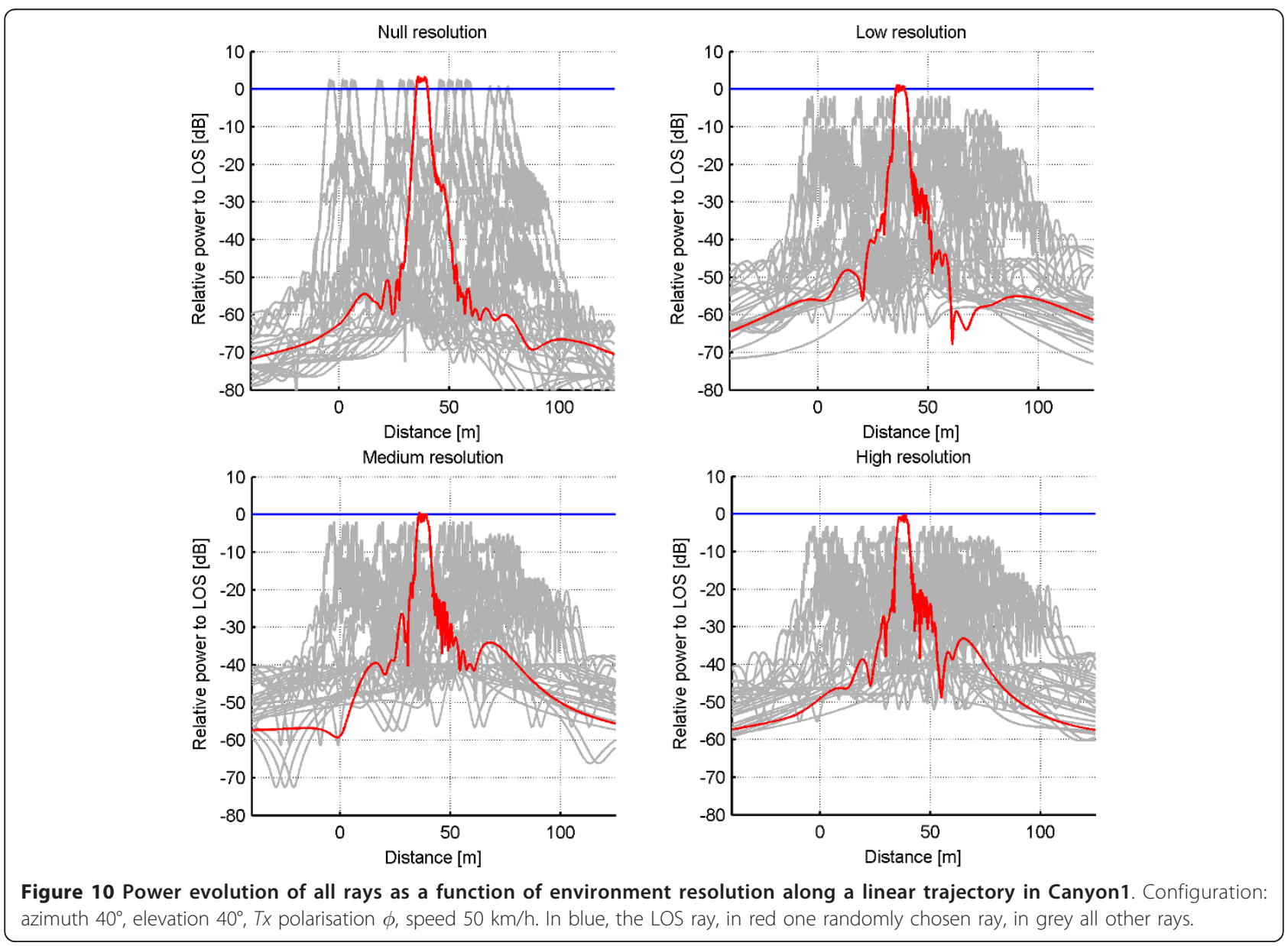



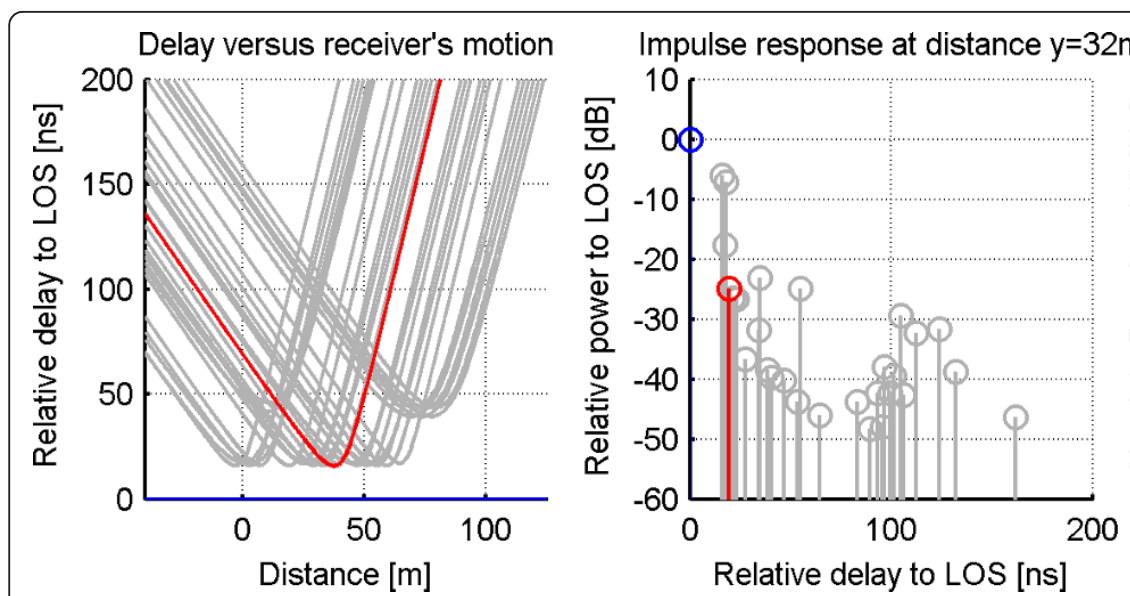

Doppler versus receiver's motion

Figure 11 Channel response parameters for a linear trajectory in Canyon 1 with high resolution. Left diagram presents the evolution of the delays of all echoes along the receiver route. Centre diagram presents the impulse response in power delay space at a specific position $y=$ $32 \mathrm{~m}$. Right diagram presents the evolution of the Doppler of all echoes along the receiver route. Configuration, azimuth $40^{\circ}$, elevation $40^{\circ}$, $T x$ polarisation $\phi$, speed $50 \mathrm{~km} / \mathrm{h}$. In blue, the LOS ray, in red one randomly chosen ray, in grey all other rays.

are also noticeable. Note that the considered red echo is the same as in Figure 10.

In order to model the wideband channel, the major assumption made in this simplified approach is the fact that each canonical building gives rise to one single ray. This approach induces discontinuities since buildings are reduced to one scattering point. This assumption comes from the MoM tool ELSEM3D we used, whose outputs are only the EM fields. A solution would have been to divide each façade into smaller pieces and record their EM fields. Using the same reradiating principle as in Section 3.1, we could have obtained 64 rays per façade instead of 1 , leading to more than 1,500 rays for Canyon1 in the high-resolution case. However, the GNSS receiver is a software model and is not adapted to process such amount of data in a reasonable time. Even more, a statistical analysis based on numerous cases would not be possible. Furthermore, this assumption is a reasonable one considering the spatial resolution of a standard $20 \mathrm{MHz}$ bandwidth system is $15 \mathrm{~m}$. This assumption is conservative since typical GNSS receivers use bandwidths between 10 and $2 \mathrm{MHz}$. In our simulated scenarios, the maximum distance between two points from the same canonical building is $7 \mathrm{~m}$, smaller than system's spatial resolution.

\subsection{Influence of environment on navigation devices}

Here, the influence of the employed environment resolution is analysed from a GNSS receiver point of view, considering the pseudo-range error as the key parameter and using the simplified wideband channel model previously described. The pseudo-range to a satellite represents the estimated distance between receiver and satellite. The pseudo-range error represents the bias induced by multipath and is the difference between the estimated and the real distance. In the following, the pseudo-range error parameter will be analysed as a function of the environment resolution for 81 different configurations ( 9 incidences, 3 speeds, 3 urban canyons). We introduced one further parameter called the relative error, which represents the offset between the pseudorange error for the simplified resolutions with respect to the high resolution.

\subsubsection{Influence of resolution and speed}

The first part of the analysis presents the influence of the environment resolution on navigation devices when the direct LOS signal is not attenuated. One example is presented in Figure 12 to illustrate a typical LMS configuration with $40^{\circ}$ elevation and $40^{\circ}$ azimuth. This figure presents, from top to bottom, the narrowband MP scattered power relative to LOS, the absolute pseudo-range error and the relative pseudo-range error with respect to that for the high-resolution representation.

A first remark concerns the configuration in Figure 12 and others not presented in the figure. It has been observed that the receiver can be sensitive to a particular wave combination resulting in punctual errors and small discontinuities visible around $y=25 \mathrm{~m}$ for the high-resolution case and $y=40 \mathrm{~m}$ for the low-resolution case on the pseudo-range error diagram. This phenomenon does not seem dependent on the chosen resolution. The consequence of this is a slight shift in the relative error, bottom diagram, while other resolutions are not affected in the same way. However, these irregularities are negligible when extracting statistical parameters.

From the narrowband analysis, it has been shown how, for typical LMS configurations, the total MP 


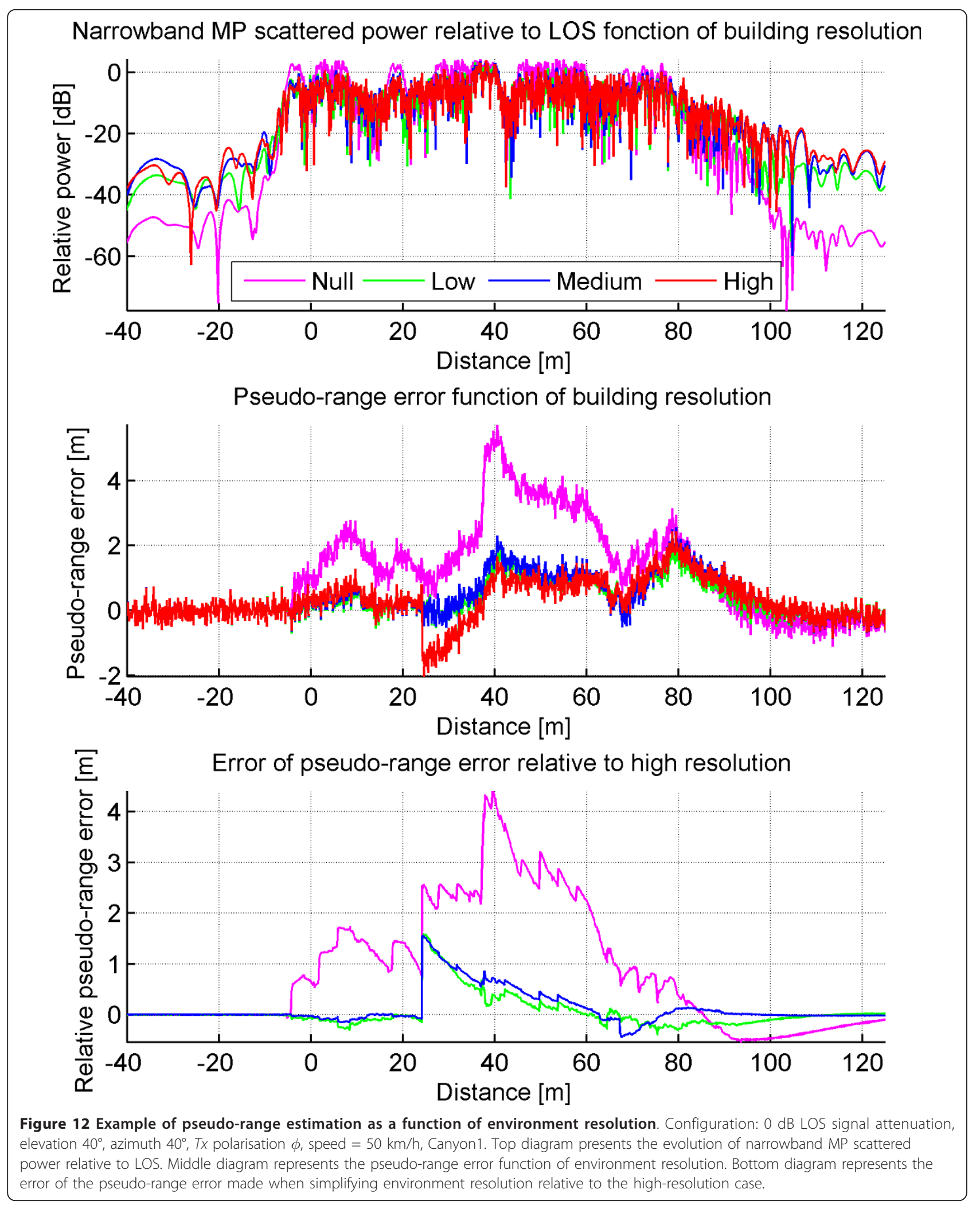


scattered power is usually overestimated. This phenomenon was particularly pronounced for null resolution. With a wideband approach, the same observation has been made. For typical LMS incidences, strong echoes are present for the null resolution resulting in high and punctual biases visible from 40 to $60 \mathrm{~m}$ in Figure 12 . Contrary to the medium and low resolutions, which show good agreement with respect to the high-resolution case, the null resolution is not suitable for typical LMS configurations.

To get a better overview and generalise the observations made in Figure 12, statistical results are next presented. For all the 81 scenarios, the mean and standard deviation error of the relative pseudo-range error have been extracted in the canyon's reflection zone, from 0 to $85 \mathrm{~m}$ in the Canyon 1 case. Figure 13 presents the cdf of both error parameters. As visible here, the mean and standard deviation error for the medium and the low resolution are good approximations with respect to the high resolution. The null resolution has critical limitations with strong mean errors.

Figure 14 presents a parametric plot considering the \{Mean;Std\} error couple, showing the influence of two parameters: environment resolution and receiver speed. The speed parameter is here taken into account since it has an important influence on the integration process taking place in the receiver. From the analysis of Figure 9 , it has been shown that slow motion was the most critical case. From the analysis of Figure 12, it has been shown that the null resolution is not suitable to represent the environment in the GNSS context. Removing

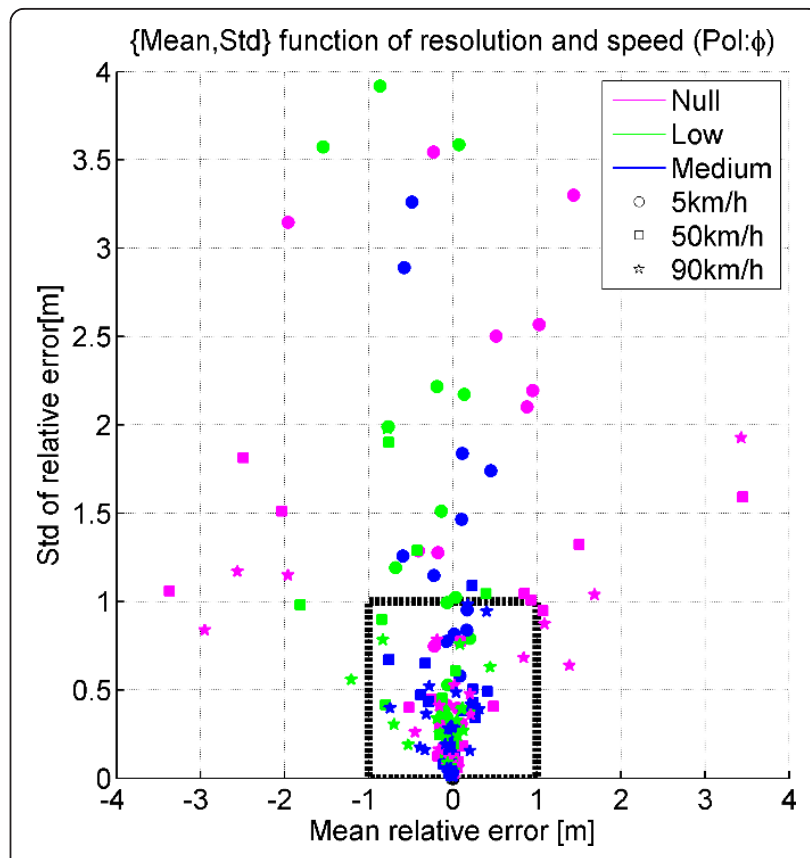

Figure 14 Parametric representation of the influence of resolution and speed on \{Mean, Std\}error couple.

both cases from Figure 14, the null resolution (pink) and the slow motion (circles), all other cases are in the dashed line black square. This square represents a mean error in the interval of $\pm 1 \mathrm{~m}$ and standard deviation error inferior to $1 \mathrm{~m}$. We can consider all these configurations as acceptable simplifications for the analysis of GNSS systems when LOS is not attenuated.
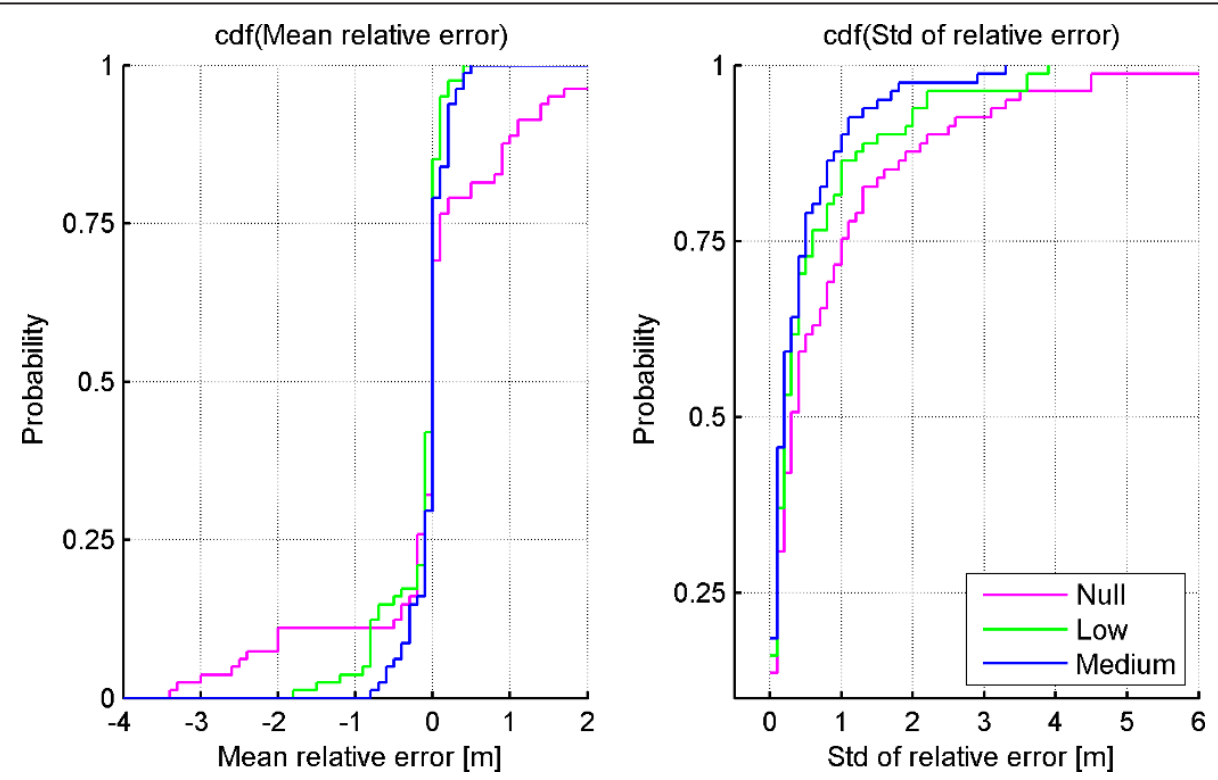

Figure 13 Influence of environment resolution on pseudo-range error for unblocked LOS signal (0 dB attenuation). Left diagram presents cdf of mean error. Right diagram presents cdf of standard error. 


\subsubsection{Influence of incidence}

In a second step, the influence of the incidence angle is here analysed. As a remainder of Section 2.3, incidences are grouped into two categories: typical LMS configurations which represent all incidences with elevation $\leq 40^{\circ}$ and azimuth $\leq 40^{\circ}$ and extreme LMS configurations which represent all other incidences with elevation $>40^{\circ}$ or azimuth $>40^{\circ}$. Figure 15 illustrates one extreme LMS incidence case with $80^{\circ}$ elevation and $80^{\circ}$ azimuth using the same representation as Figure 12. Figure 15 is representative of the fact that for extreme incidences pseudorange errors are very small $( \pm 0.5 \mathrm{~m})$ and are noise-like. Furthermore, relative errors are almost null in the range of $\pm 0.1 \mathrm{~m}$. In general, for all configurations, it has been noted that pseudo-range errors occur when the MP scattered power is larger than $-15 \mathrm{~dB}$ relative to the LOS signal. This explains the low impact of MP for the extreme incidences. In this case, the environment resolution has no impact compared to the typical geometrical LMS configurations.

To confirm this statement, statistics are presented in Figure 16. They have been extracted considering the null resolution. This case is the most conservative one since the null resolution has the largest spread in the mean error as shown in Figure 13. From Figure 16, it can be observed how all extreme cases have a mean error almost equal to zero and standard deviation below $1 \mathrm{~m}$, in agreement with Figure 15. This confirms that for extreme LMS incidences even the null resolution produces acceptable results.

\subsubsection{Influence of LOS attenuation}

In the previous scenarios, the direct signal was not attenuated. To extend the domain of validity of this analysis and better take into account real cases, attenuation on the direct signal has been investigated. Two attenuations are considered: -5 and $-20 \mathrm{~dB}$ corresponding to tree shadowing and building blockage. It is assumed that the direct signal is the only component affected by this attenuation. The consequence is that the margin between the MP echoes and the direct signal is reduced. It may even occur that some echoes are stronger than the direct signal.

Simulations realised for $-5 \mathrm{~dB}$ show similar results to those for no direct signal attenuation. Thus, for this case, the same conclusions are applicable. However, results differ when considering a $-20-\mathrm{dB}$ attenuation as presented in Figure 17. Here, the MP scattered power is about $20 \mathrm{~dB}$ higher than the attenuated direct signal. When a long blockage event occurs, the receiver tends to track the strongest echo. This results in all resolutions being biased in the same way. As visible in Figure 17, the pseudo-range error is about 6 $\mathrm{m}$, representative of the distance between the receiver trajectory and the first row of buildings, situated at 4 $\mathrm{m}$ from the receiver's route. We can also notice fluctuations around $y=20 \mathrm{~m}$ due to the second row of buildings, situated $10 \mathrm{~m}$ from the receiver. A similar behaviour is particularly visible from $y=70 \mathrm{~m}$ to the end of receiver's route. From point $y=70 \mathrm{~m}$ onwards specular reflections are not possible. While the receiver continues down its route, the DLL keeps tracking the strongest diffuse contribution coming from last building corner. The result is that pseudo-range error increases until the diffuse contribution falls down at around $y=90 \mathrm{~m}$. Then, the attenuated direct signal becomes predominant over the MP, and the receiver recovers track of the LOS signal. Finally, looking at the first half of the pseudo-range error diagram in the figure, for high and medium resolutions, the GNSS receiver seems to be affected by MP earlier than for low and null resolutions. The explanation is that when the LOS signal is attenuated, any small multipath contribution becomes noticeable by the receiver. Since scattering is almost absent in the low and null resolutions, both resolutions are affected later by MP, and only in the reflection zone. The medium and high resolutions show a longer impact, before and after. Not presented in the figure, the above observations are even more visible for extreme azimuths where the corner effect from the details does not remain negligible when comparing the null and low resolutions to the medium and high ones.

Figure 18 shows the statistics on the influence of the resolution when the direct signal is attenuated by $20 \mathrm{~dB}$. Contrary to unblocked case, the environment resolution has a significant impact on the receiver's performance.

\section{Conclusion}

In this article, a rigorous method has been followed to evaluate the influence of small scatterers present in complex environments on LMS and GNSS propagation channels. The goal of the study was to better understand what level of simplification is acceptable when describing complex environments to be used as inputs to EM tools. A possible application of this study could be simplifying the representation of buildings to use asymptotic methods and, particularly, PO-based methods. The whole analysis has been split into a narrowband and wideband study of this problem.

The whole analysis has been conducted with a rigorous MoM-based EM tool, namely ELSEM3D, which permits to accurately reproduce the scattering phenomenon from small objects/façade features, without any object size limitations. ELSEM3D has been used to precisely simulate the scattered EM field around canonical buildings described in various levels of detail.

The main conclusions from the narrowband analysis are listed below. These conclusions are applicable both 


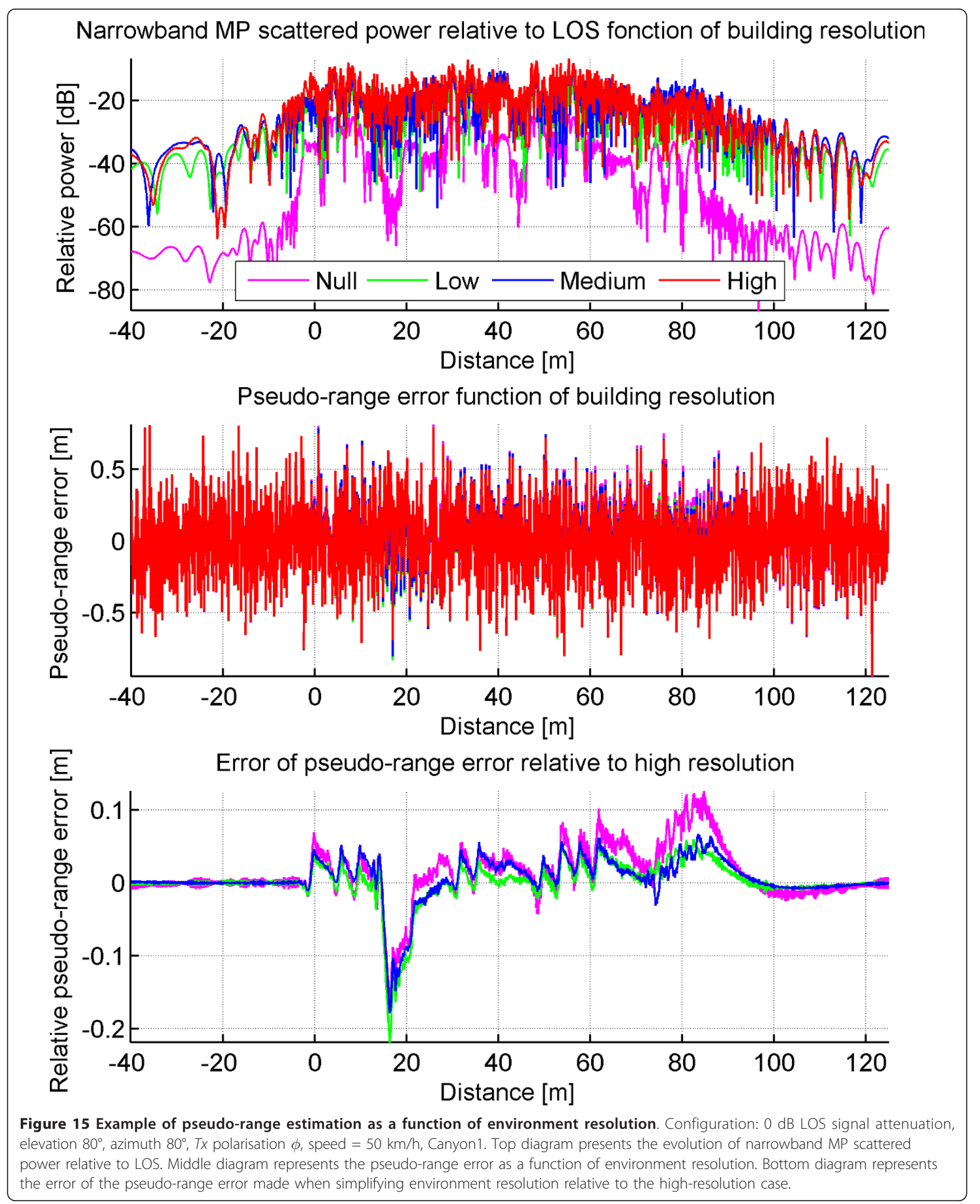



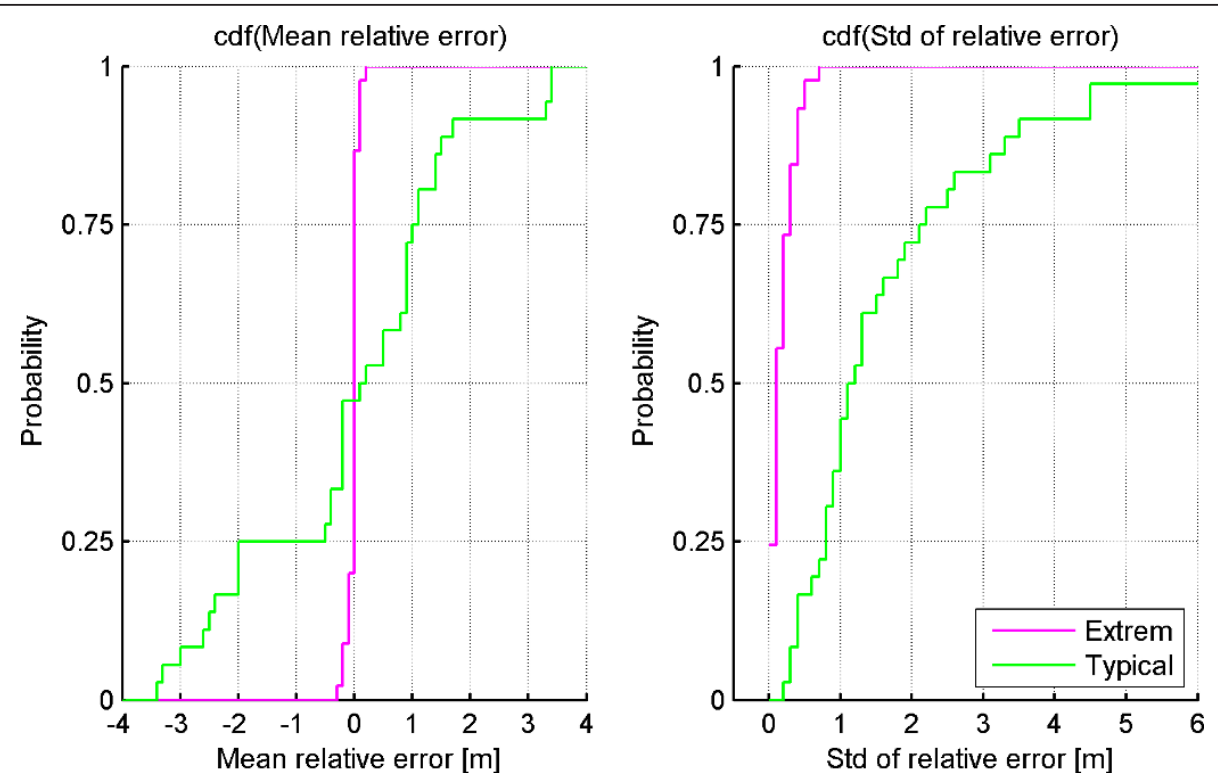

Figure 16 Influence of extreme incidences on pseudo-range error for null resolution for unblocked LOS signal. Left diagram presents cdf of mean error. Right diagram presents cdf of standard error.

to communication and navigation systems.

- Null resolution: the scattering phenomenon is not reproduced. It results in an MP scattered power overestimation for "typical" LMS incidences and in underestimation for "extreme" LMS incidences. The null resolution is almost comparable to an on/ off reflection model with significant transition effects.

- Low resolution seems acceptable if slightly corrected by an empirical offset on the mean MP scattered power and reducing the signal dynamic.

- Medium resolution is the best compromise since high resolution is not applicable at city scale.

As a recommendation, modelling the environment with details in the range of one wavelength and larger is advised for slow motion and extreme incidences. For other cases, only details larger than the wavelength could be sufficient to represent the environment if empirical adjustments are applied to the obtained MP scattered power. From our experience, $+2 \mathrm{~dB}$ would be a typical offset value. The canonical plate representation is hardly acceptable even for a first estimation of the MP scattered power.

The second part of the analysis was focused on a wideband approach. The influence of the environment resolution has been assessed using pseudo-range estimations with a simplified GNSS receiver model. The conclusions are the following:
- For cases with unblocked direct signal: low resolution is sufficient to describe the channel behaviour through the GNSS receiver. For extreme incidences, even the null resolution can be used since the MP is not predominant compared to LOS signal. This conclusion remains valid for low attenuations of the direct signal, e.g. $-5 \mathrm{~dB}$.

- For cases where the direct signal suffers high attenuation: the low resolution is not sufficient any more, especially when a transition occurs between the specular and non-specular regions. A more detailed environment representation is then necessary, at least around transition states. We advise to use medium resolution in this case.

As a recommendation, for wideband applications and particularly for satellite navigation, details larger than the wavelength are sufficient to model the environment if the satellite is visible or slightly masked. For strong shadowing, the environment should contain smaller details in the range of the wavelength. For particular applications where precise results are needed, a particular attention should be paid when the GNSS receiver transits from the specular to the non-specular zone. Note that pedestrian and slow motion applications are the most critical ones since the integration process does not mitigate deep fades. Also for the slow motion case, environment sampling effects can be visible on GNSS performances when reducing buildings to point scatterers. 


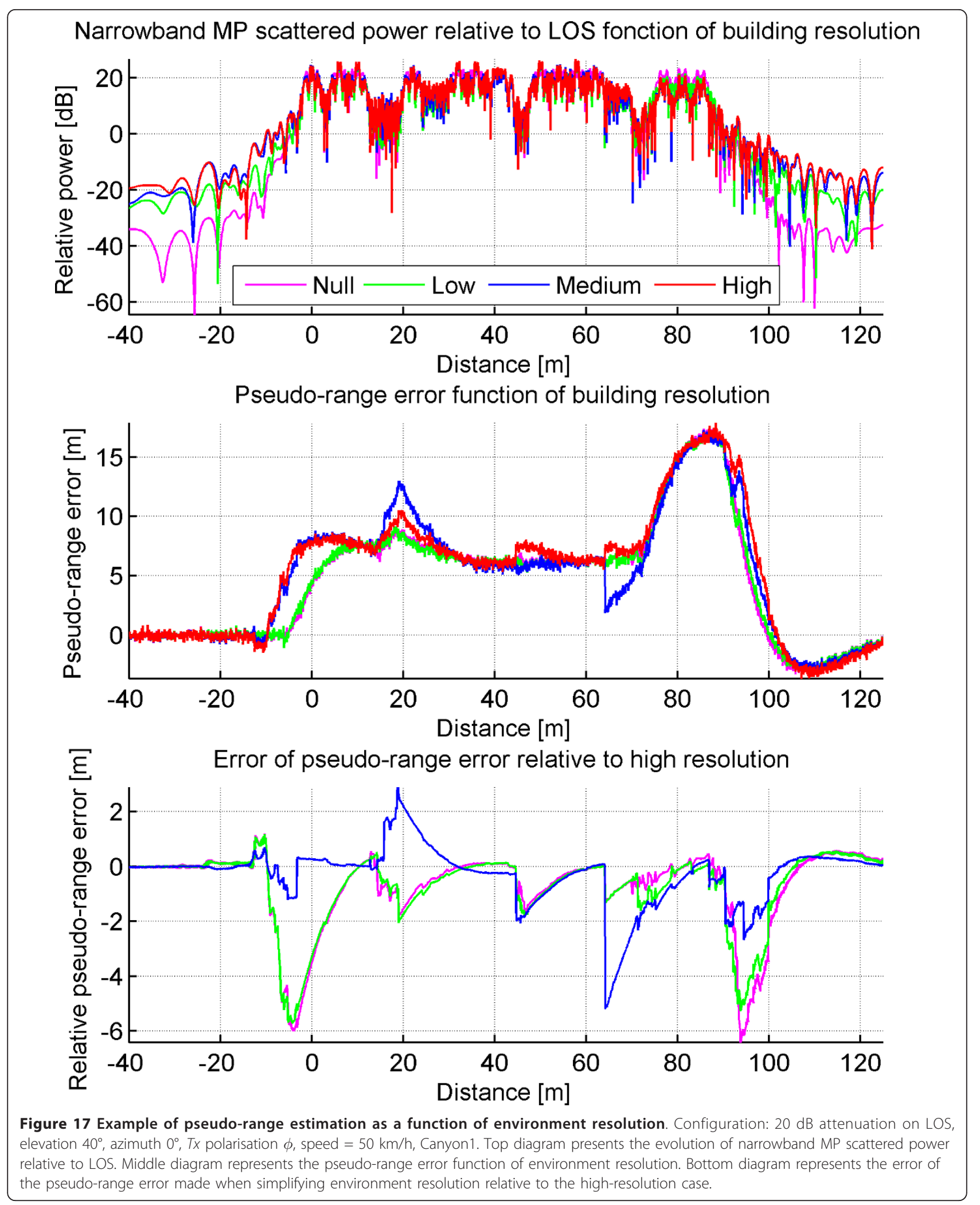



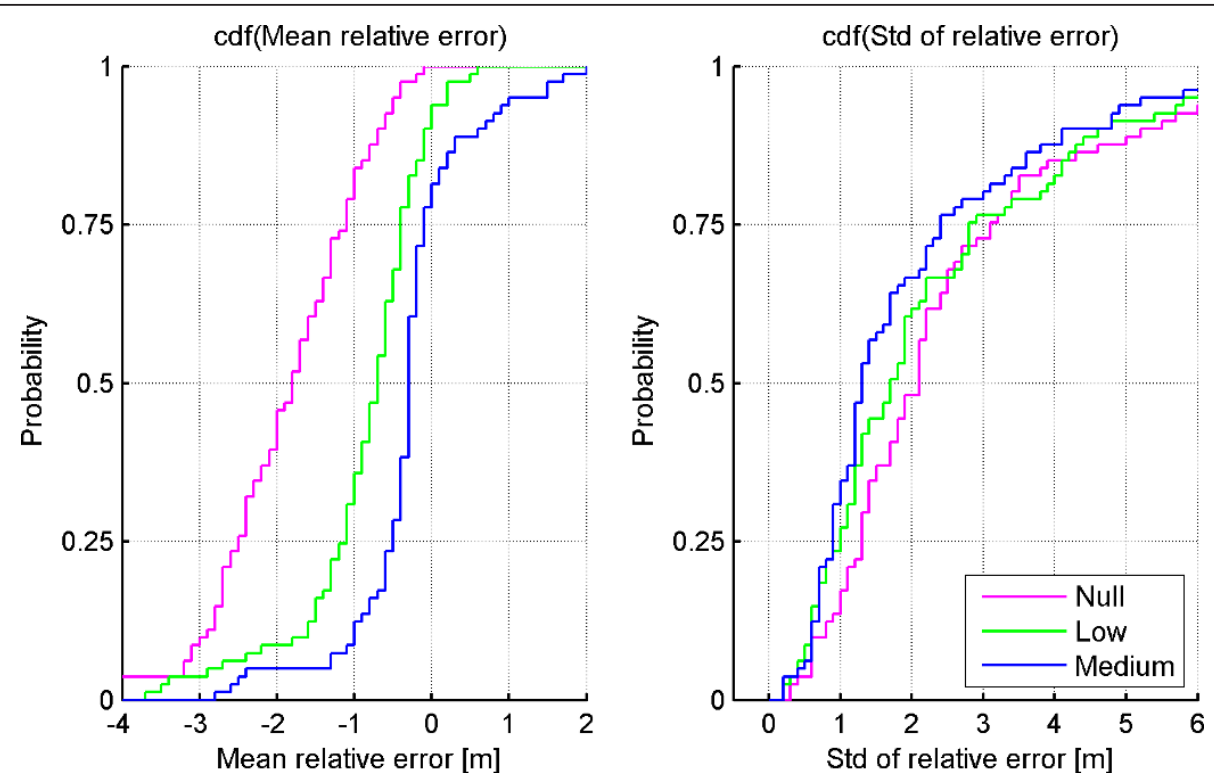

Figure 18 Influence of environment resolution on pseudo-range error for a $\mathbf{2 0 ~ d B}$ attenuated LOS signal. Left diagram presents cdf of mean error. Right diagram presents cdf of standard error.

Finally, it should be pointed out that the resolution has more impact on the MP scattered power estimation than on the pseudo-range estimation.

This study could be extended and completed by a similar work on the influence of dielectric materials chosen when modelling complex environments.

\section{Abbreviations}

cdf: cumulative density function; DLL: delay lock loop; EM: ElectroMagnetic; GNSS: Global Navigation Satellite System; GPS: Global Positioning System; LMS: Land Mobile Satellite; LOS: line of sight; MoM: Method of Moments; MP: MultiPaths; NLOS: non-line of sight; PEC: perfect electric conductor; PLL: phase lock loop; PO: physical optics; PRN: pseudo random noise; RCS: radar cross section.

\section{Author details}

'ONERA - The French Aerospace Lab., 31055 Toulouse, France ${ }^{2}$ CNES Toulouse, 18 Avenue Edouard Belin, 31400 Toulouse, France ${ }^{3}$ Universidad de Vigo, 36.310 Vigo, Spain ${ }^{4}$ Thales Alenia Space, 26 Avenue Jean François Champollion, 31100 Toulouse, France ${ }^{5}$ ISAE Campus Supaéro, 10 Avenue Edouard Belin, 31055 Toulouse, France

\section{Competing interests}

The authors declare that they have no competing interests.

Received: 5 May 2011 Accepted: 15 March 2012

Published: 15 March 2012

\section{References}

1. FP Fontan, S Martínez, B Sanmartín, C Enjamio, P Mariño, F Machado, An enhanced Markov chain based model for the narrowband LMS channel in built-up areas. Int J Satellite Commun Netw. 23, 111-128 (2005). doi:10.1002/sat.809

2. L Diaz, T Milligan, Engineering Using Physical Optics. Practical CAD Techniques and Software (Artech House, Inc Norwood, MA, USA, 1996)

3. M Ait-lghil, J Lemorton, F Pérez-Fontán, F Lacoste, V Gobin, G Artaud, C Bourga, M Bousquet, Numerical analysis of the impact of building face features on LMS channel modeling. Antennas and Propagation (EUCAP), Proceedings of the 5th European Conference on 3566-3570 (2011)

4. C Loo, A statistical model for a land mobile satellite link. IEEE Trans Veh Technol. 34, 122-127 (1985)

5. Castanet $L$ (ed.), Influence of the variability of the Propagation Channel on Mobile, Fixed Multimedia and Optical Satellite Communications (Shaker Verlag, Aachen, 2008)

6. FP Fontan, M Vazquez-Castro, CE Cabado, JP Garcia, E Kubista, Statistical modeling of the LMS channel. IEEE Trans Veh Technol. 50, 1549-1567 (2001). doi:10.1109/25.966585

7. C Oestges, SR Saunders, D Vanhoenacker-Janvier, Physical statistical modelling of the land mobile satellite channel based on ray tracing. IEEE Proc Microwaves Antennas Propag. 146, 45-49 (1999). doi:10.1049/ipmap:19990144

8. A Lehner, Multipath channel modelling for satellite navigation systems. PhD thesis, Erlangen, Germany (2007)

9. ITU, ITU-R P.681-7 Propagation data required for the design of Earth-space land mobile telecommunication systems. ITU (2009)

10. A Abele, FP Fontan, M Bousquet, P Valtr, J Lemorton, F Lacoste, E Corbel, A new physical-statistical model of the land mobile satellite propagation channel. Antennas and Propagation (EUCAP), 2010 Proceedings of the Fourth European Conference on 1-5 (2010)

11. P Valtr, FP Fontan, A Abele, Propagation modelling in virtual environments, characterization of mobile propagation channel. Antennas and Propagation (EuCAP), 2009 Proceedings of the Thrid European Conference on 3678-3681 (2009)

12. Cost action 255 - Radiowave Propagation Modelling for SatCom Services at Ku-Band and Above, Final report (2002). ISBN 92-9092-608-2

13. A Barka, P Soudais, T Volpert, Scattering from $3 D$ cavities with a plug and play numerical scheme combining IE, PDE and modal techniques. Antennas and Propagation, IEEE Transactions on. 48(5), 704-712 (2000). doi:10.1109/ 8.855488

14. P Soudais, H Steve, F Dubois, Scattering from several test-objects computed by 3-D hybrid IE/PDE methods. Antennas and Propagation, IEEE Transactions on. 47(4), 646-653 (1999). doi:10.1109/8.768803

15. P Carlos, P Yannis, V Rodolphe, C Pierre, Sensitivity of the MIMO Channel Characterization to the Modeling of the Environment. Antennas and Propagation, IEEE Transactions on. 57(4), 1218-1227 (2009)

16. B Ouattara, Développement d'une approche radar pour l'étude des réflexions sur les bâtiments et l'analyse des irrégularités de façade. PhD thesis, Université Paris-Est, France (2010) 
17. E Kaplan, C Hegarty, Understanding GPS: Principles and Applications, 1st edn. (Artech House, Inc Norwood, MA, USA, 1996)

18. BW Parkinson, J Spilker, in Global Positioning System: Theory and Application, vol. 1. (AIAA, Washington, 1996)

doi:10.1186/1687-1499-2012-110

Cite this article as: Ait-lghil et al: Simplifying the propagation

environment representation for LMS channel modelling. EURASIP Journal on Wireless Communications and Networking 2012 2012:110.

\section{Submit your manuscript to a SpringerOpen ${ }^{\circ}$ journal and benefit from:}

- Convenient online submission

- Rigorous peer review

- Immediate publication on acceptance

- Open access: articles freely available online

- High visibility within the field

- Retaining the copyright to your article

Submit your next manuscript at $\gg$ springeropen.com 